\title{
Intrinsic Metric Formulas on Some Self-Similar Sets via the Code Representation
}

\author{
Melis Güneri ${ }^{1}$ and Mustafa Saltan ${ }^{2, *(D)}$ \\ 1 Department of Mathematics, Bilecik Şeyh Edebali University, Bilecik 11230, Turkey; \\ mguneri3.mg@gmail.com \\ 2 Department of Mathematics, Eskişehir Technical University, Eskişehir 26470, Turkey \\ * Correspondence: mustafasaltan@eskisehir.edu.tr; Tel.: +90-222-335-0580
}

Received: 4 February 2019; Accepted: 22 March 2019; Published: 25 March 2019

\begin{abstract}
In recent years, intrinsic metrics have been described on various fractals with different formulas. The Sierpinski gasket is given as one of the fundamental models which defined the intrinsic metrics on them via the code representations of the points. In this paper, we obtain the explicit formulas of the intrinsic metrics on some self-similar sets (but not strictly self-similar), which are composed of different combinations of equilateral and right Sierpinski gaskets, respectively, by using the code representations of their points. We then express geometrical properties of these structures on their code sets and also give some illustrative examples.
\end{abstract}

Keywords: Sierpinski gasket; self-similar sets; code representation; intrinsic metric

MSC: 28A80; 51F99

\section{Introduction}

Fractal geometry is one of the most remarkable developments in mathematics in recent years. Since fractals are central to apprehending a wide array of chaotic and nonlinear systems, they have many applications in physics, chemistry, biology, computer science, engineering, economics and so on (for details, see [1-4]). One of the common properties of fractals is self-similarity. A geometric shape is self-similar if there is a point such that every neighborhood of the point contains a copy of the entire shape and, if it is self-similar at every point, then it is called strictly self-similar. For example, the Sierpinski gasket, the Cantor set, the Koch curve and the Sierpinski carpet are strictly self-similar sets (for details, see [5]). There have been different studies on these fractals since the 1970s. Defining the intrinsic metric on these sets is one of them [6-11]. In [12], the intrinsic metric on the code set of the Sierpinski gasket, which is one of the instructive examples of strictly self-similar sets, is formulated by the code representations of its points. Due to this metric formula, important geometrical and topological properties of the Sierpinski gasket are expressed by the code sets, the number of geodesics are determined and the code representations of points are classified according to the number of geodesics (for details, see [13-15]). As seen in these studies, defining the intrinsic metrics by using the code representations of the points on fractals provides some facilities for different works. Hence, our aim is to increase such examples in the literature.

In this paper, we define two new fractals which are self-similar but are not strictly self-similar and then we determine the code representations of points on these fractals. To this end, we first constitute a model by using three classical Sierpinski gaskets with edge length one. We also express code representations of the points on this structure. In Theorem 2, we define the intrinsic metric on this structure and present some geometrical properties on its code set in Propositions 3 and 4 . Finally, we formulate the intrinsic metric on a second fractal obtained by two right Sierpinski gaskets 
in Proposition 5. As seen in Examples 1 and 3, one of the advantages of these intrinsic metric formulas is to calculate the distance between the points on these sets easily.

\section{The Intrinsic Metric on the Code Set of the Sierpinski Gasket}

It is well known that the intrinsic metric on a set $X$ is defined as

$$
d_{\text {int }}(x, y)=\inf \{\delta \mid \delta \text { is the length of a rectifiable curve in } X \text { joining } x \text { and } y\}
$$

for $x, y \in X$ (see [16]). By using the code representations of the points, to express the intrinsic metrics on fractals is a valuable problem, but it is not easy to obtain these formulas for every self-similar set. Therefore, the Sierpinski gasket $(S)$ is regarded as one of the fundamental models which define the intrinsic metric formulas on fractals (for details, see [12]). It is well known that the attractor of the iterated function system $\left\{\mathbb{R}^{2} ; f_{0}, f_{1}, f_{2}\right\}$ such that

$$
\begin{aligned}
& f_{0}(x, y)=\left(\frac{x}{2}+\frac{p_{0}}{2}, \frac{y}{2}+\frac{p_{1}}{2}\right), \\
& f_{1}(x, y)=\left(\frac{x}{2}+\frac{q_{0}}{2}, \frac{y}{2}+\frac{q_{1}}{2}\right), \\
& f_{2}(x, y)=\left(\frac{x}{2}+\frac{r_{0}}{2}, \frac{y}{2}+\frac{r_{1}}{2}\right)
\end{aligned}
$$

is the Sierpinski gasket with the vertices $P=\left(p_{0}, p_{1}\right), Q=\left(q_{0}, q_{1}\right)$ and $R=\left(r_{0}, r_{1}\right)$. That is,

$$
S=\bigcup_{i=0}^{2} f_{i}(S)
$$

Note that, if these coefficients are taken as $p_{0}=p_{1}=q_{1}=0, q_{0}=1, r_{0}=\frac{1}{2}$ and $r_{1}=\frac{\sqrt{3}}{2}$, then the attractor of the iterated function system is the classical Sierpinski gasket (see Figure 1). In the case of $p_{0}=p_{1}=q_{1}=r_{0}=0, q_{0}=1$ and $r_{1}=1$, the so-called right Sierpinski gasket is then obtained as the attractor of the iterated function system.

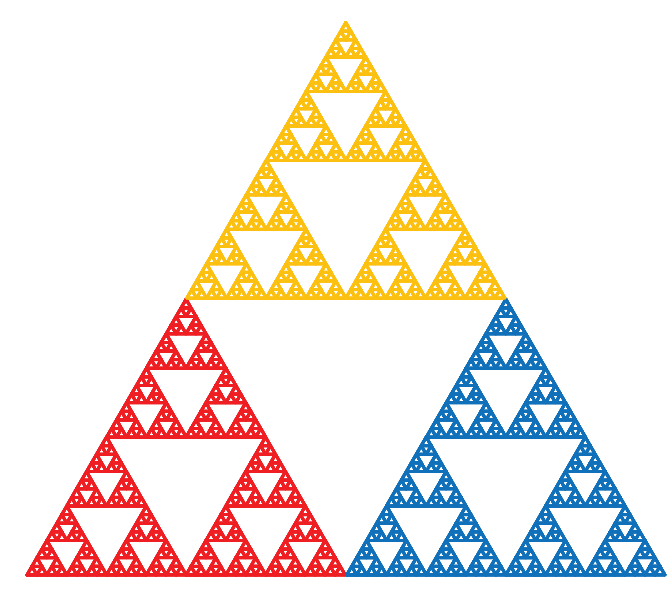

Figure 1. The classical Sierpinski gasket.

The intrinsic metric on the code set of the scalene Sierpinski gasket with the vertices $P=\left(p_{0}, p_{1}\right), Q=\left(q_{0}, q_{1}\right)$ and $R=\left(r_{0}, r_{1}\right)$ is formulated in [15] as follows:

Let $a_{i}=b_{i}$ for $i=1,2, \ldots, k-1$ and $a_{k} \neq b_{k}$, where $a_{i}, b_{i} \in\{0,1,2\}$ for $i=1,2,3, \ldots$ If the code representations points $A$ and $B$ on this set are $a_{1} a_{2} \ldots a_{k-1} a_{k} a_{k+1} \ldots$ and $b_{1} b_{2} \ldots b_{k-1} b_{k} b_{k+1} \ldots$, respectively, then the intrinsic metric formula is defined as follows: 
Theorem 1 ([15], Theorem 1). Let $a_{k} \neq c_{k} \neq b_{k}$ and $c_{k} \in\{0,1,2\}$ and

$$
\kappa= \begin{cases}|P Q|, & \left(a_{k}=0, b_{k}=1\right) \text { or }\left(a_{k}=1, b_{k}=0\right), \\ |P R|, & \left(a_{k}=0, b_{k}=2\right) \text { or }\left(a_{k}=2, b_{k}=0\right), \\ |Q R|, & \left(a_{k}=1, b_{k}=2\right) \text { or }\left(a_{k}=2, b_{k}=1\right) .\end{cases}
$$

Then, the formula

$$
d(A, B)=\min \left\{\sum_{i=k+1}^{\infty} \frac{\alpha_{i}+\beta_{i}}{2^{i}}, \frac{\kappa}{2^{k}}+\sum_{i=k+1}^{\infty} \frac{\gamma_{i}+\delta_{i}}{2^{i}}\right\}
$$

such that

$$
\begin{gathered}
\alpha_{i}=\left\{\begin{array}{cl}
0, & a_{i}=b_{k}, \\
|P Q|, & \left(a_{i}=0, b_{k}=1\right) \text { or }\left(a_{i}=1, b_{k}=0\right), \\
|P R|, & \left(a_{i}=0, b_{k}=2\right) \text { or }\left(a_{i}=2, b_{k}=0\right), \\
|Q R|, & \left(a_{i}=1, b_{k}=2\right) \text { or }\left(a_{i}=2, b_{k}=1\right),
\end{array} \quad \beta_{i}=\left\{\begin{array}{cc}
0, & b_{i}=a_{k}, \\
|P Q|, & \left(b_{i}=0, a_{k}=1\right) \text { or }\left(b_{i}=1, a_{k}=0\right), \\
|P R|, & \left(b_{i}=0, a_{k}=2\right) \text { or }\left(b_{i}=2, a_{k}=0\right), \\
|Q R|, & \left(b_{i}=1, a_{k}=2\right) \text { or }\left(b_{i}=2, a_{k}=1\right),
\end{array}\right.\right. \\
\gamma_{i}=\left\{\begin{array}{cl}
0, & a_{i}=c_{k}, \\
|P Q|, & \left(a_{i}=0, c_{k}=1\right) \text { or }\left(a_{i}=1, c_{k}=0\right), \\
|P R|, & \left(a_{i}=0, c_{k}=2\right) \text { or }\left(a_{i}=2, c_{k}=0\right), \\
|Q R|, & \left(a_{i}=1, c_{k}=2\right) \text { or }\left(a_{i}=2, c_{k}=1\right),
\end{array} \quad \delta_{i}=\left\{\begin{array}{cc}
0, & b_{i}=c_{k}, \\
|P Q|, & \left(b_{i}=0, c_{k}=1\right) \text { or }\left(b_{i}=1, c_{k}=0\right), \\
|P R|, & \left(b_{i}=0, c_{k}=2\right) \text { or }\left(b_{i}=2, c_{k}=0\right), \\
|Q R|, & \left(b_{i}=1, c_{k}=2\right) \text { or }\left(b_{i}=2, c_{k}=1\right),
\end{array}\right.\right.
\end{gathered}
$$

gives the length of the shortest path between the points A and B on the scalene Sierpinski gasket.

Remark 1. The intrinsic metric formula on the right Sierpinski gasket is defined as follows:

$$
d(A, B)=\min \left\{\sum_{i=k+1}^{\infty} \frac{\alpha_{i}+\beta_{i}}{2^{i}}, \frac{\kappa}{2^{k}}+\sum_{i=k+1}^{\infty} \frac{\gamma_{i}+\delta_{i}}{2^{i}}\right\},
$$

such that

$$
\begin{aligned}
& \alpha_{i}=\left\{\begin{array}{cl}
0, & a_{i}=b_{k} \\
\sqrt{2}, & \left(a_{i}=1, b_{k}=2\right) \text { or }\left(a_{i}=2, b_{k}=1\right), \quad \beta_{i}=\left\{\begin{array}{cl}
0, & b_{i}=a_{k}, \\
\sqrt{2}, & \left(b_{i}=1, a_{k}=2\right) \text { or }\left(b_{i}=2, a_{k}=1\right) \\
1, & \text { otherwise, }
\end{array}\right. \text { otherwise, }
\end{array}\right. \\
& \gamma_{i}=\left\{\begin{array}{cl}
0, & a_{i}=c_{k}, \\
\sqrt{2}, & \left(a_{i}=1, c_{k}=2\right) \text { or }\left(a_{i}=2, c_{k}=1\right), \quad \delta_{i}=\left\{\begin{array}{cl}
0, & b_{i}=c_{k}, \\
\sqrt{2}, & \left(b_{i}=1, c_{k}=2\right) \text { or }\left(b_{i}=2, c_{k}=1\right) \\
1, & \text { otherwise, }
\end{array}\right. \text { otherwise, }
\end{array}\right.
\end{aligned}
$$

and

$$
\kappa=\left\{\begin{array}{cl}
\sqrt{2}, & \left(a_{k}=1, b_{k}=2\right) \text { or }\left(a_{k}=2, b_{k}=1\right), \\
1, & \text { otherwise. }
\end{array}\right.
$$

The intrinsic metric formula on the classical Sierpinski gasket is obtained by

$$
d(A, B)=\min \left\{\sum_{i=k+1}^{\infty} \frac{\alpha_{i}+\beta_{i}}{2^{i}}, \frac{1}{2^{k}}+\sum_{i=k+1}^{\infty} \frac{\gamma_{i}+\delta_{i}}{2^{i}}\right\}
$$


where

$$
\begin{array}{cc}
\alpha_{i}= \begin{cases}0, & a_{i}=b_{k}, \\
1, & a_{i} \neq b_{k},\end{cases} & \beta_{i}= \begin{cases}0, & b_{i}=a_{k} \\
1, & b_{i} \neq a_{k},\end{cases} \\
\gamma_{i}=\left\{\begin{array}{ll}
0, & a_{i} \neq a_{k} \text { and } a_{i} \neq b_{k}, \\
1, & \text { otherwise, }
\end{array} \quad \delta_{i}= \begin{cases}0, & b_{i} \neq b_{k} \text { and } b_{i} \neq a_{k}, \\
1, & \text { otherwise }\end{cases} \right.
\end{array}
$$

(for details, see $[12,15])$.

\section{The Code Representations of Points on the Sierpinski Propellers}

Let us first consider identical classical Sierpinski gaskets whose colors are red, yellow, blue, black and purple. As seen in Figure 2, we combine $m$ copies of the identical classical Sierpinski gaskets (for $m=2,3,4,5$, respectively) at one touching point $T$. Note that these shapes are similar to a propeller. Thus, we call these new structures as the Sierpinski propeller (briefly SP). Since the fractal dimension of the Sierpinski gasket is $\frac{\ln 3}{\ln 2}$, the fractal dimensions of the Sierpinski propellers are also $\frac{\ln 3}{\ln 2}$. Moreover, the areas of these sets are 0 .
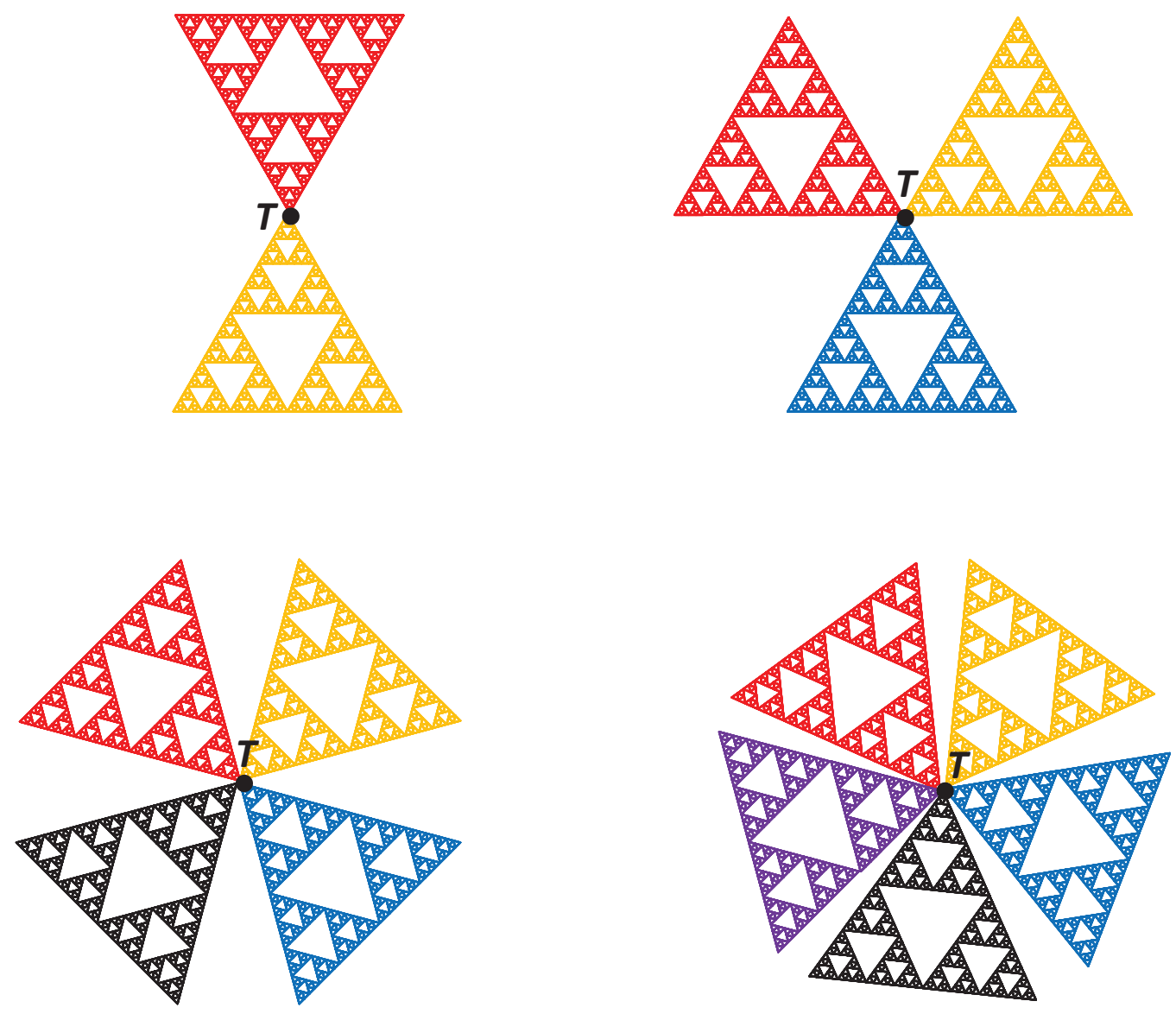

Figure 2. The classical Sierpinski propellers.

The Sierpinski gasket is a strictly self-similar. Although the Sierpinski propellers are self-similar, they are not strictly self-similar. It can easily be seen that $T$ is a special point since every neighborhood of this point contains the scaled copies of the Sierpinski propellers (see Figure 3 and Proposition 4). 


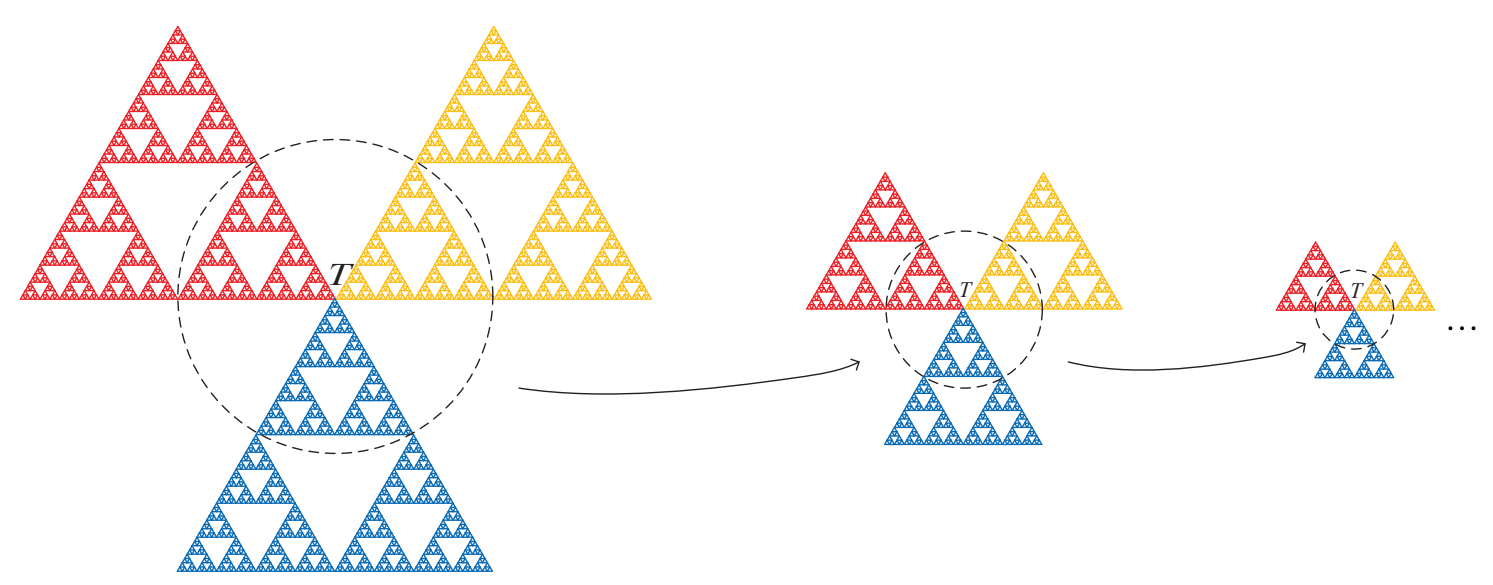

Figure 3. The scaled copies of the classical Sierpinski propeller for $m=3$.

In this section, we only express the code representations of points on the classical Sierpinski propeller for $m=3$ and we define the intrinsic metric on the code set of this fractal in Section 4 . The intrinsic metric formulas and the code sets of the classical Sierpinski propellers for $m=2,4,5$ can be similarly obtained. Note that the intrinsic metric formula can be expressed more simply for $m=3$ when a different code representation is used (see Remark 4). However, the following method can be more convenient for the proofs in cases of $m=4$ and $m=5$ (the numbers of the code representations of the point $T$ are 4 and 5 , respectively).

Let us denote the red Sierpinski gasket, the yellow Sierpinski gasket and the blue Sierpinski gasket of the Sierpinski propeller by $S P_{\widetilde{0}}, S P_{\widetilde{1}}$ and $S P_{\widetilde{2}}$, respectively. It is obvious that

$$
S P_{\widetilde{0}} \cap S P_{\widetilde{1}} \cap S P_{\widetilde{2}}=T
$$

and

$$
S P_{\widetilde{0}} \cup S P_{\widetilde{1}} \cup S P_{\widetilde{2}}=S P .
$$

Let us state the left-bottom part, the right-bottom part and the upper part of $S P_{a_{0}}$ by $S P_{a_{0} 0}, S P_{a_{0} 1}$ and $S P_{a_{0} 2}$, respectively, where $a_{0} \in\{\widetilde{0}, \widetilde{1}, \widetilde{2}\}$. The smaller triangular pieces of $S P$ are denoted by $S P_{a_{0} a_{1} a_{2} \ldots a_{k}}$, where $a_{i} \in\{0,1,2\}$ and $i=1,2, \ldots, k$. We thus obtain

$$
S P_{a_{0}} \supset S P_{a_{0} a_{1}} \supset S P_{a_{0} a_{1} a_{2}} \supset S P_{a_{0} a_{1} a_{2} a_{3}} \supset \ldots \supset S P_{a_{0} a_{1} a_{2} \ldots a_{n}} \supset \ldots
$$

and for a sequence of nested sets $S P_{a_{0}}, S P_{a_{0} a_{1}}, S P_{a_{0} a_{1} a_{2}}, S P_{a_{0} a_{1} a_{2} a_{3}}, \ldots$, the Cantor intersection theorem states that the infinite intersection of these sets contains exactly one point:

$$
\bigcap_{k=0}^{\infty} S P_{a_{0} a_{1} a_{2} \ldots a_{k}}=\{A\}
$$

We denote the point $A \in S P$ by $a_{0} a_{1} a_{2} \ldots a_{n} \ldots$ where $a_{0} \in\{\widetilde{0}, \widetilde{1}, \widetilde{2}\}$ and $a_{n} \in\{0,1,2\}$ for $n=1,2, \ldots$

The following proposition shows that the points on the Sierpinski propeller have the code representations different from the code representations of points on the Sierpinski gasket:

Proposition 1. The Sierpinski propeller has points whose numbers of the code representations are 1, 2 or 3 . Moreover, the point $T$ is the unique point whose number of the code representations is 3. 
Proof. $T$ is the unique point which has three code representations such that $\widetilde{0} 111 \ldots, \widetilde{1} 000 \ldots$ and $\widetilde{2} 222 \ldots$ since all of the set sequences

$$
\begin{aligned}
& S P_{\widetilde{0}}, S P_{\tilde{0}_{1}}, S P_{\tilde{0}_{11}}, S P_{0111}, \ldots \\
& S P_{\widetilde{1}}, S P_{\widetilde{1}_{0}}, S P_{\widetilde{1}_{00}}, S P_{\widetilde{1000}_{00}}, \ldots
\end{aligned}
$$

and

$$
S P_{\widetilde{2}}, S P_{\widetilde{2} 2}, S P_{\widetilde{2} 22}, S P_{\widetilde{2} 222}, \ldots
$$

contain the point $T$. Note that, for $A \neq T$, if $A$ is the intersection point of any two sub-triangles in the same level of $S_{a_{0} a_{1} a_{2} \ldots a_{k}}$, then $A$ has two different representations such that $a_{0} a_{1} a_{2} \ldots a_{k} \beta \alpha \alpha \alpha \alpha \ldots$ and $a_{0} a_{1} a_{2} \ldots a_{k} \alpha \beta \beta \beta \beta \ldots$ where $\alpha, \beta \in\{0,1,2\}$. Otherwise, the code representation of $A$ is unique.

In Figure 4, it can be seen that the set of code representations of points on the red Sierpinski gasket is

$$
S P_{\widetilde{0}}=\left\{\widetilde{0} a_{1} a_{2} a_{3} \ldots \mid a_{i} \in\{0,1,2\}\right\},
$$

the set of code representations of points on the yellow Sierpinski gasket is

$$
S P_{\widetilde{1}}=\left\{\widetilde{1} a_{1} a_{2} a_{3} \ldots \mid a_{i} \in\{0,1,2\}\right\}
$$

and the set of code representations of points on the blue Sierpinski gasket is

$$
S P_{\widetilde{2}}=\left\{\widetilde{2} a_{1} a_{2} a_{3} \ldots \mid a_{i} \in\{0,1,2\}\right\} .
$$

Generally, the sub-triangles of $S_{\sigma}$ for $\sigma=a_{0} a_{1} a_{2} \ldots a_{k-1}$ are expressed as

$$
S P_{\sigma}=\left\{\sigma a_{k} a_{k+1} a_{k+2} \ldots \mid a_{i} \in\{0,1,2\}, i=k, k+1, k+2, \ldots\right\} .
$$

In addition, the code sets of the Sierpinski propellers are

$$
\left\{\widetilde{0} 1 \ldots 1 a_{k+1} \ldots \mid a_{i} \in\{0,1,2\}\right\} \cup\left\{\widetilde{1} 0 \ldots 0 a_{k+1} \ldots \mid a_{i} \in\{0,1,2\}\right\} \cup\left\{\widetilde{2} 2 \ldots 2 a_{k+1} \ldots \mid a_{i} \in\{0,1,2\}\right\},
$$

where $i=k+1, k+2, k+3 \ldots$ for $k=1,2,3, \ldots$

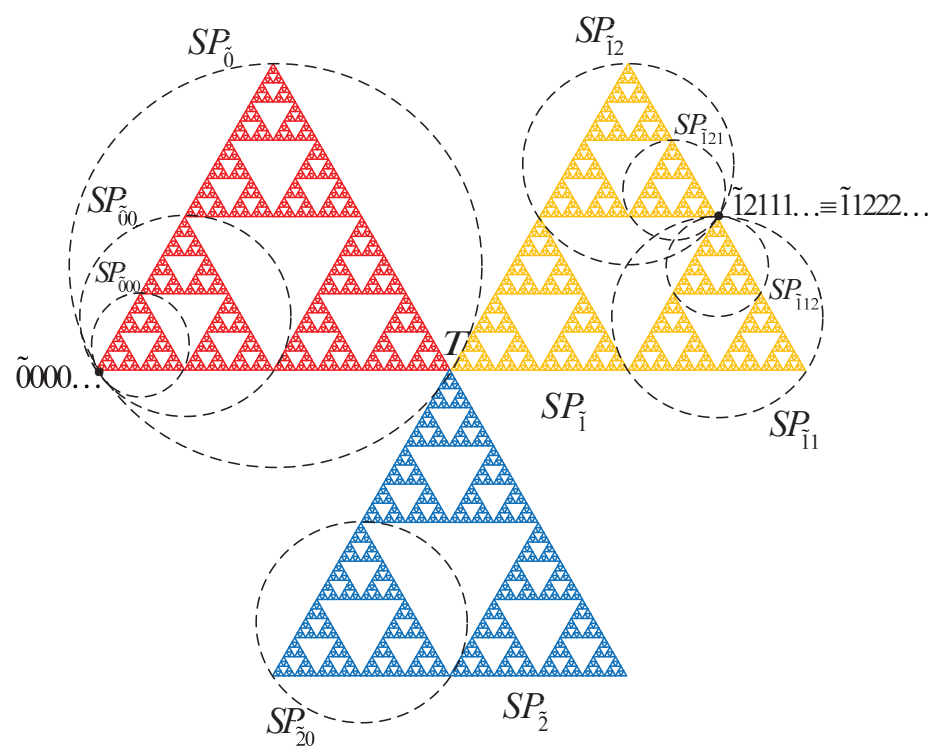

Figure 4. Some code sets of the Sierpinski propeller. 


\section{The Construction of the Intrinsic Metric on the Code Set of SP}

In the following theorem, we formulate the intrinsic metric on SP by using the code representations of its points.

Theorem 2. Suppose that the code representations of the points $A$ and $B$ on the Sierpinski propeller are $a_{0} a_{1} a_{2} \ldots a_{k-1} a_{k} a_{k+1} \ldots$ and $b_{0} b_{1} b_{2} \ldots b_{k-1} b_{k} b_{k+1} \ldots$ respectively such that $a_{i}=b_{i}$ for $i=1,2, \ldots, k-1$ and $a_{k} \neq b_{k}$ where $a_{0}, b_{0} \in\{\widetilde{0}, \widetilde{1}, \widetilde{2}\}$ and $a_{i}, b_{i} \in\{0,1,2\}$ for $i=1,2,3, \ldots$

(i) If $a_{0} \neq b_{0}$, then the shortest distance between $A$ and $B$ is determined by

$$
d(A, B)=\sum_{i=1}^{\infty} \frac{\widetilde{\alpha}_{i}+\widetilde{\beta}_{i}}{2^{i}}
$$

such that

$$
\begin{aligned}
& \widetilde{\alpha}_{i}=\left\{\begin{array}{ll}
0, & a_{i}=1 \\
1, & a_{i} \neq 1
\end{array}, \quad \widetilde{\beta}_{i}=\left\{\begin{array}{ll}
0, & b_{i}=0 \\
1, & b_{i} \neq 0
\end{array} \quad \text { if }\left(a_{0}=\widetilde{0}, b_{0}=\widetilde{1}\right),\right.\right. \\
& \widetilde{\alpha}_{i}=\left\{\begin{array}{ll}
0, & a_{i}=1 \\
1, & a_{i} \neq 1
\end{array}, \quad \widetilde{\beta}_{i}=\left\{\begin{array}{ll}
0, & b_{i}=2 \\
1, & b_{i} \neq 2
\end{array} \quad \text { if }\left(a_{0}=\widetilde{0}, b_{0}=\widetilde{2}\right),\right.\right. \\
& \widetilde{\alpha}_{i}=\left\{\begin{array}{ll}
0, & a_{i}=0 \\
1, & a_{i} \neq 0
\end{array}, \quad \widetilde{\beta}_{i}=\left\{\begin{array}{ll}
0, & b_{i}=2 \\
1, & b_{i} \neq 2
\end{array}, \quad \text { if }\left(a_{0}=\widetilde{1}, b_{0}=\widetilde{2}\right) .\right.\right.
\end{aligned}
$$

(ii) If $a_{0}=b_{0}$, then Equation (4) gives the desired distance.

Proof. (i) Suppose that $A$ and $B$ are two different points of the Sierpinski propeller with the code representations $a_{0} a_{1} a_{2} \ldots a_{n} \ldots$ and $b_{0} b_{1} b_{2} \ldots b_{n} \ldots$, respectively, where $a_{0} \neq b_{0}$. It is clear that the shortest path between $A$ and $B$ must pass through the point $T$ since $S P_{a_{0}} \cap S P_{b_{0}}=\{T\}$.

Firstly, let us consider the case of $a_{0}=\widetilde{0}$ and $b_{0}=\widetilde{1}$ (the other cases are done similarly). If $a_{1}=0$ or $a_{1}=2$, then the shortest path between $A$ and $T$ must pass through the points $S P_{\widetilde{0} 0} \cap S P_{\widetilde{0} 1}$ or $S P_{\widetilde{0} 1} \cap S P_{\widetilde{0} 2}$, respectively. These points have the code representations $\widetilde{0} 1000 \ldots$ (equivalently $\widetilde{0} 0111 \ldots$ ) or $\widetilde{0} 1222 \ldots$ (equivalently $\widetilde{0} 2111 \ldots$ ), respectively. Furthermore, $T$ and $S P_{\widetilde{0} 0} \cap S P_{\widetilde{0} 1}$ (similarly $T$ and $S P_{\widetilde{0} 1} \cap S P_{\widetilde{0} 2}$ ) are the points which are the vertices in the sub-triangle $S P_{\widetilde{0} 1}$ of the Sierpinski gasket. Hence, the length of the shortest path between these points is $\frac{1}{2}$. We compute the length of the shortest path between $A$ and $T$ as $\frac{1}{2}$ if the code representations of $A$ are $\widetilde{0} 1000 \ldots$ (equivalently $\widetilde{0} 0111 \ldots$ ) or $\widetilde{0} 1222 \ldots$ (equivalently $\widetilde{0} 2111 \ldots$.). If $a_{1}=1$ and the code representations of $A$ are not $\widetilde{0} 1000 \ldots$ and $\widetilde{0} 1222 \ldots$, then the length of the shortest path between $A$ and $T$ is less than $\frac{1}{2}$. Consequently, in (5), we get $\widetilde{\alpha}_{1}=1$ if $a_{1}=0$ or $a_{1}=2$ and we get $\widetilde{\alpha}_{1}=0$ if $a_{1}=1$.

Now, consider $a_{1}=0$ and let us take $a_{2}=0$ or $a_{2}=2$ (see Figure 5). Then, the shortest path between $A$ and $S P_{\widetilde{0} 0} \cap S P_{\widetilde{0} 1}$ must pass through $S P_{\widetilde{0} 00} \cap S P_{\widetilde{0} 01}$ or $S P_{\widetilde{0} 01} \cap S P_{\widetilde{0} 02}$, respectively. Note that these intersections have the code representations $\widetilde{0} 01000 \ldots$ (equivalently $\widetilde{0} 00111 \ldots$. .) or $\widetilde{0} 01222 \ldots$ (equivalently $\widetilde{0} 02111 \ldots$ ), respectively. In addition, $\widetilde{0} 0111 \ldots$ and $S P_{\widetilde{0} 00} \cap S P_{\widetilde{0} 01}$ (similarly $\widetilde{0} 0111 \ldots$ and $\left.S P_{\widetilde{0} 01} \cap S P_{\widetilde{0} 02}\right)$ are the points that are the vertices in the sub-triangle $S P_{\widetilde{0} 01}$ of the Sierpinski gasket. Thus, the length of the shortest path between these points is $\frac{1}{2^{2}}$. We compute the length of the shortest path between $A$ and $T$ as $\frac{1}{2}+\frac{1}{2^{2}}$ if the code representations of $A$ are $\widetilde{0} 00111 \ldots$ or $\widetilde{0} 02111 \ldots$. If $a_{2}=1$ and the code representations of $A$ are not $\widetilde{0} 01000 \ldots$ and $\widetilde{0} 01222 \ldots$, then the length of the shortest path between $A$ and $T$ is less than $\frac{1}{2}+\frac{1}{2^{2}}$. Consequently, in (5), we get $\widetilde{\alpha}_{2}=1$ if $a_{2}=0$ or $a_{2}=2$ and we get $\widetilde{\alpha}_{2}=0$ if $a_{2}=1$. Generally, for $i=1,2,3, \ldots$, we obtain $\widetilde{\alpha}_{i}=1$ if $a_{i}=0$ or $a_{i}=2$ and we get $\widetilde{\alpha}_{i}=0$ if 
$a_{i}=1$. A similar procedure is also valid for the computation of the length of the shortest path between the points $T$ and $B$.

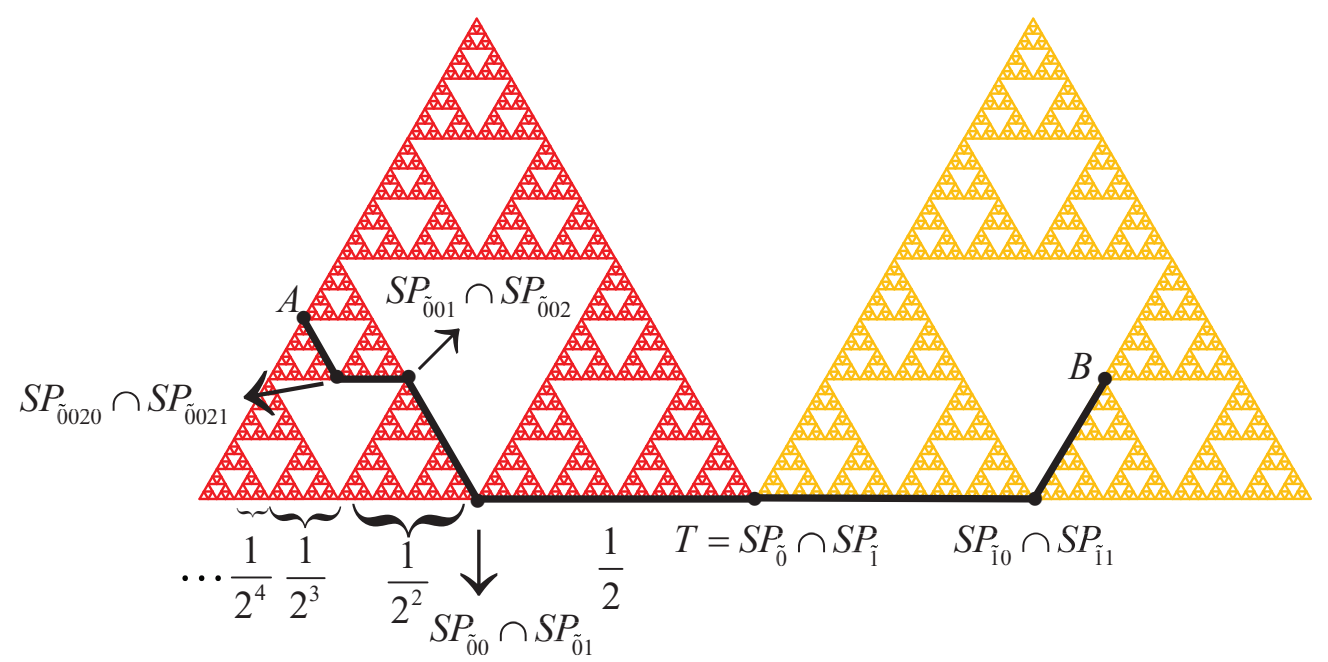

Figure 5. One of the shortest paths between $A$ and $B$ on the Sierpinski propeller for $a_{0}=\widetilde{0}$ and $b_{0}=\widetilde{1}$.

Remark 2. The point $T$ has three code representations such as $\widetilde{0} 111 \ldots, \widetilde{1} 000 \ldots$ and $\widetilde{2} 222 \ldots$. Due to Formula (5), it is easily seen that the distance (which is computed by using different codes of this point) is 0 . Thus, using a similar method given in [12], the following proposition can be proven.

Proposition 2. The metric $d$ defined in Theorem 2 does not depend on the choice of the code representations of the points.

Example 1. Assume that $A$ and $B$ are the points of SP whose code representations are $1010202 \ldots$ and 2010101..., respectively. For the computation of $d(A, B)$, we must use Formula (8) owing to the fact that the first terms of the code representations of $A$ and $B$ are $\widetilde{1}$ and $\widetilde{2}$, respectively. Thus, we get

$$
\widetilde{\alpha}_{i}=0 \text { for } i=2,4,6, \ldots \widetilde{\alpha}_{i}=1, \text { for } i=1,3,5, \ldots
$$

and

$$
\widetilde{\beta}_{i}=1 \text { for } i=1,2,3, \ldots
$$

It follows that

$$
\begin{aligned}
d(A, B)=\sum_{i=1}^{\infty} \frac{\widetilde{\alpha}_{i}+\widetilde{\beta}_{i}}{2^{i}} & =\frac{0+1}{2}+\frac{1+1}{2^{2}}+\frac{0+1}{2^{3}}+\frac{1+1}{2^{4}}+\cdots \\
& =\frac{1}{2}+\frac{2}{2^{2}}+\frac{1}{2^{3}}+\frac{2}{2^{4}}+\cdots \\
& =\frac{4}{3} .
\end{aligned}
$$

Proposition 3. If $d$ is the intrinsic metric defined in (5) on $S P$, then

$$
\operatorname{diam}(S P)=\max \{d(A, B) \mid A, B \in S P\}=2 .
$$

Moreover, $d(A, B)=2$ if and only if the code representations of $A$ are an element of the set $\left\{\widetilde{0} a_{1} a_{2} a_{3} \ldots \mid a_{i} \in\right.$ $\{0,2\}\}$ and the code representations of $B$ are an element of the sets $\left\{\tilde{1} b_{1} b_{2} b_{3} \ldots \mid b_{i} \in\{1,2\}\right\}$ or $\left\{\widetilde{2} b_{1} b_{2} b_{3} \ldots \mid b_{i} \in\{0,1\}\right\}$, or if the code representations of $A$ are an element of the set $\left\{\tilde{1} a_{1} a_{2} a_{3} \ldots \mid a_{i} \in\right.$ 
$\{1,2\}\}$ and the code representations of $B$ are an element of the sets $\left\{\widetilde{0} b_{1} b_{2} b_{3} \ldots \mid b_{i} \in\{0,2\}\right\}$ or $\left\{\widetilde{2} b_{1} b_{2} b_{3} \ldots \mid b_{i} \in\{0,1\}\right\}$.

Proof. For the computation of the maximum value of the distances between any two points $A$ and $B$ of $S P, \widetilde{\alpha}_{i}$ and $\widetilde{\beta_{i}}$ must be 1 for $i=1,2,3, \ldots$ (see Formula (5)). Thus, if $a_{0}=\widetilde{0}$ and $b_{0}=\widetilde{1}$, then $a_{i} \neq 1$ and $b_{i} \neq 0$ or if $a_{0}=\widetilde{0}$ and $b_{0}=\widetilde{2}$, then $a_{i} \neq 1$ and $b_{i} \neq 2$ for $i=1,2,3, \ldots$ (see Formulas (6) and (7)). In addition, if $a_{0}=\widetilde{1}$ and $b_{0}=\widetilde{2}$, then $a_{i} \neq 0$ and $b_{i} \neq 2$ (see Formula (8)). In these cases, we obtain

$$
\begin{aligned}
d(A, B)=\sum_{i=1}^{\infty} \frac{\widetilde{\alpha}_{i}+\widetilde{\beta}_{i}}{2^{i}} & =\frac{1+1}{2}+\frac{1+1}{2^{2}}+\frac{1+1}{2^{3}}+\frac{1+1}{2^{4}}+\cdots \\
& =1+\frac{1}{2}+\frac{1}{2^{2}}+\frac{1}{2^{3}}+\cdots \\
& =2 .
\end{aligned}
$$

Proposition 4. For $n=1,2,3, \ldots$, the closed discs of radii $\frac{1}{2^{n}}$ with center $T$ are the scaled copy of Sierpinski propeller. Especially, if $n=0$, then $D(T, 1)=S P$. In addition, the code sets of circles of radii $\frac{1}{2^{n}}$ with center T are

$$
\left\{\widetilde{0} 1 \ldots 1 a_{n+1} \ldots \mid a_{i} \in\{0,2\}\right\} \cup\left\{\widetilde{1} 0 \ldots 0 a_{n+1} \ldots \mid a_{i} \in\{1,2\}\right\} \cup\left\{\widetilde{2} 2 \ldots 2 a_{n+1} \ldots \mid a_{i} \in\{0,1\}\right\} .
$$

Proof. We first compute the code representations of the points $A$ satisfying $d(A, T)=\frac{1}{2^{n}}$. Without loss of generality, we take $\widetilde{2} 222 \ldots$ as the code representation of $T$. Let the code representation of $A$ be $a_{0} a_{1} a_{2} \ldots$ where $a_{0} \in\{\widetilde{0}, \widetilde{1}, \widetilde{2}\}$ and $a_{i} \in\{0,1,2\}$ for $i=1,2,3, \ldots$

Case 1: Suppose that $a_{0}=\widetilde{0}$. Since the terms of code representation of $T$ (for $\mathrm{i}=1,2,3, \ldots$ ) are 2, we get $\widetilde{\beta}_{i}=0$ for $i=1,2,3, \ldots$ In addition, $a_{i}$ must be 1 for $i \leq n-1$ (see Formula (7)). Otherwise, we compute $d(A, T) \geq \frac{1}{2^{n-1}}$. Now, consider that $a_{i}$ are 1 for all $i=1,2,3, \ldots, n$. This means that $\widetilde{\alpha_{i}}=0$ for $i=1,2,3, \ldots, n$. If $a_{i} \neq 1$ for $i=n+1, n+2, n+3, \ldots$, then we obtain $\widetilde{\alpha_{i}}=1$. Therefore, we compute

$$
d(A, T)=\sum_{i=n+1}^{\infty} \frac{1+0}{2^{i}}=\frac{1}{2^{n}}
$$

and this shows that $\left\{\widetilde{0} 1 \ldots 1 a_{n+1} a_{n+2} \ldots \mid a_{i} \in\{0,2\}\right\}$ is one of the parts of the set of the code representations of $A$ satisfying $d(A, T)=\frac{1}{2^{n}}$ for $a_{0}=\widetilde{0}$.

From the construction above, it can be easily seen that, if $d(A, T) \leq \frac{1}{2^{n}}$ for $a_{0}=\widetilde{0}$, then $\left\{\widetilde{0} 1 \ldots 1 a_{n+1} a_{n+2} \ldots \mid a_{i} \in\{0,1,2\}\right\}$ is one of the parts of the set of the code representations of $A$.

Case 2: Let $a_{0}=\widetilde{1}$. By using Formula (8) and following a method similar to Case 1, we obtain that $\left\{\widetilde{1} 0 \ldots 0 a_{n+1} a_{n+2} \ldots \mid a_{i} \in\{1,2\}\right\}$ is one of the parts of the set of the code representations of $A$ satisfying $d(A, T)=\frac{1}{2^{n}}$. Moreover, we compute if $d(A, T) \leq \frac{1}{2^{n}}$ for $a_{0}=\widetilde{1}$, then $\left\{\widetilde{1} 0 \ldots 0 a_{n+1} a_{n+2} \ldots \mid a_{i} \in\{0,1,2\}\right\}$ is one of the parts of the set of the code representations of $A$.

Case 3: Assume that $a_{0}=\widetilde{2}$. Then, the points $A$ and $T$ are in the same Sierpinski gasket. Applying Formula (4), we obtain that $\left\{\widetilde{2} 2 \ldots 2 a_{n+1} a_{n+2} \ldots \mid a_{i} \in\{0,1\}\right\}$ and $\left\{\widetilde{2} 2 \ldots 2 a_{n+1} a_{n+2} \ldots \mid a_{i} \in\{0,1,2\}\right\}$ are one of the parts of the set of the code representations of $A$ satisfying $d(A, T)=\frac{1}{2^{n}}$ and $d(A, T) \leq \frac{1}{2^{n}}$, respectively. 
Thus, the unions of sets obtained in Cases 1, 2 and 3 give us the code representations of $A$ satisfying $d(A, T)=\frac{1}{2^{n}}$ and $d(A, T) \leq \frac{1}{2^{n}}$. That is,

$$
\begin{aligned}
S(A, T)= & \left\{\widetilde{0} 1 \ldots 1 a_{n+1} a_{n+2} \ldots \mid a_{i} \in\{0,2\}\right\} \cup\left\{\widetilde{1} 0 \ldots 0 a_{n+1} a_{n+2} \ldots \mid a_{i} \in\{1,2\}\right\} \\
& \cup\left\{\widetilde{2} 2 \ldots 2 a_{n+1} a_{n+2} \ldots \mid a_{i} \in\{0,1\}\right\}
\end{aligned}
$$

and

$$
\begin{aligned}
D(A, T)= & \left\{\widetilde{0} 1 \ldots 1 a_{n+1} a_{n+2} \ldots \mid a_{i} \in\{0,1,2\}\right\} \cup\left\{\widetilde{1} 0 \ldots 0 a_{n+1} a_{n+2} \ldots \mid a_{i} \in\{0,1,2\}\right\} \\
& \cup\left\{\widetilde{2} 2 \ldots 2 a_{n+1} a_{n+2} \ldots \mid a_{i} \in\{0,1,2\}\right\} .
\end{aligned}
$$

Example 2. Consider the point A of SP which has the code representation 0000 .... Obviously, the code set of the closed disc of radius $\frac{1}{2}$ with center $A$ is

$$
D\left(\widetilde{0} 000 \ldots, \frac{1}{2}\right)=\left\{\widetilde{0} 0 a_{2} a_{3} a_{4} \ldots \mid a_{i} \in\{0,1,2\}\right\} .
$$

Remark 3. Proposition 4 and Example 2 show that SP is a self-similar set but not strictly self-similar.

Remark 4. In the Sierpinski propeller for $m=3$, if the red Sierpinski gasket is coded by $S P_{\widetilde{1}}$ and the yellow Sierpinski gasket is coded by $S P_{\widetilde{0}}$, then the code representations of the point $T$ are determined by $\widetilde{w} w w w . . .$, where $w \in\{0,1,2\}$. Thus, the intrinsic metric formula can be expressed by

$$
d(A, B)=\sum_{i=1}^{\infty} \frac{\widetilde{\alpha}_{i}+\widetilde{\beta}_{i}}{2^{i}}
$$

such that

$$
\widetilde{\alpha}_{i}=\left\{\begin{array}{ll}
0, & a_{i}=a_{0}, \\
1, & a_{i} \neq a_{0},
\end{array}, \quad \widetilde{\beta}_{i}= \begin{cases}0, & b_{i}=b_{0}, \\
1, & b_{i} \neq b_{0},\end{cases}\right.
$$

for $a_{0} \neq b_{0}$. Moreover, the code sets of circles of radii $\frac{1}{2^{n}}$ with center $T$ can be determined by

$$
\left\{\widetilde{a_{0}} a_{0} a_{0} \ldots a_{0} a_{n+1} \ldots \mid a_{i} \neq a_{0}, i \geq n+1\right\} .
$$

\section{The Intrinsic Metric Formula on Two Adjacent Right Sierpinski Gaskets}

By using two right Sierpinski gaskets with vertices $P, Q$ and $R$ where $|P Q|=|P R|=1$ and $|Q R|=\sqrt{2}$, we now define a second fractal which is self-similar but not strictly self-similar. Consider two identical right Sierpinski gakets whose colors of sub-triangles are red, blue and yellow, and then combine these two triangles at the point $Q$ as in Figure 6. We call this structure adjacent right Sierpinski gaskets, briefly $A S$.

Let us denote the triangle that is left of the point $Q$ by $A S_{\tilde{0}}$ and the triangle that is the right of the point $Q$ by $A S_{\tilde{1}}$. In addition, let us denote the red Sierpinski gaskets by $A S_{\widetilde{0} 0}$ and $A S_{\widetilde{1} 0}$, respectively, the blue Sierpinski gaskets by $A S_{\widetilde{0} 1}$ and $A S_{\widetilde{1} 1}$, respectively, and the yellow Sierpinski gaskets by $A S_{\widetilde{0} 2}$ and $A S_{\widetilde{1} 2}$, respectively. It is clear that

$$
A S_{\widetilde{0} 1} \cap A S_{\widetilde{1} 1}=Q
$$

and

$$
A S_{\widetilde{0}} \cup A S_{\widetilde{1}}=A S .
$$

Thus, two different code representations of $Q$ are $\widetilde{0} 111 \ldots$ and $\widetilde{1} 111 \ldots$ It is also easily seen that a point of $A S$ has either one code representation or two code representations. 


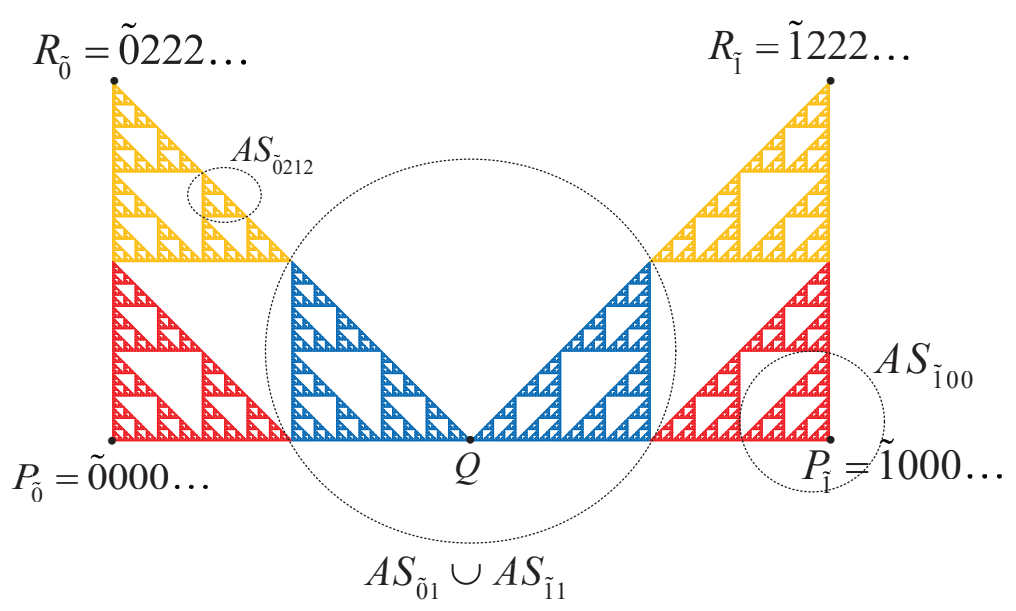

Figure 6. Some code sets and code representations of $A S$.

In the following proposition, we express the intrinsic metric on the code set of AS:

Proposition 5. Suppose that the code representations of the points $A$ and $B$ on AS are $a_{0} a_{1} a_{2} \ldots a_{k-1} a_{k} a_{k+1} \ldots$ and $b_{0} b_{1} b_{2} \ldots b_{k-1} b_{k} b_{k+1} \ldots$ respectively such that $a_{i}=b_{i}$ for $i=1,2, \ldots, k-1$ and $a_{k} \neq b_{k}$, where $a_{0}, b_{0} \in\{\widetilde{0}, \widetilde{1}\}$ and $a_{i}, b_{i} \in\{0,1,2\}$ for $i=1,2,3, \ldots$

(i) If $a_{0} \neq b_{0}$, then the shortest distance between $A$ and $B$ is determined by

$$
d(A, B)=\sum_{i=1}^{\infty} \frac{\widetilde{\alpha}_{i}+\widetilde{\beta}_{i}}{2^{i}}
$$

such that

$$
\widetilde{\alpha}_{i}=\left\{\begin{array}{rl}
0, & a_{i}=1, \\
1, & a_{i}=0, \\
\sqrt{2}, & a_{i}=2,
\end{array} \quad \widetilde{\beta}_{i}=\left\{\begin{array}{cc}
0, & b_{i}=1 \\
1, & b_{i}=0 \\
\sqrt{2}, & b_{i}=2
\end{array}\right.\right.
$$

(ii) If $a_{0}=b_{0}$, then Equation (3) gives the desired distance.

Proof. The proof is omitted since it is similar to the proof of Theorem 2.

Example 3. Let the code representation of $A$ be $\widetilde{0} 020202 \ldots$ and let the code representation of $B$ be $\widetilde{1} 101010 \ldots$. We must use Formula (12) to compute $d(A, B)$ since the first terms of the code representations of $A$ and $B$ are different. Then, we obtain

$$
\widetilde{\alpha}_{i}=1, \text { for } i=1,3,5, \ldots \widetilde{\alpha}_{i}=\sqrt{2} \text { for } i=2,4,6, \ldots
$$

and

$$
\widetilde{\beta}_{i}=0 \text { for } i=1,3,5, \ldots \widetilde{\beta}_{i}=1 \text { for } i=2,4,6, \ldots
$$

Thus, we get

$$
\begin{aligned}
d(A, B)=\sum_{i=1}^{\infty} \frac{\widetilde{\alpha}_{i}+\widetilde{\beta}_{i}}{2^{i}} & =\frac{1+0}{2}+\frac{\sqrt{2}+1}{2^{2}}+\frac{1+0}{2^{3}}+\frac{\sqrt{2}+1}{2^{4}}+\cdots \\
& =\left(\frac{1}{2}+\frac{2}{2^{3}}+\frac{1}{2^{5}}+\cdots\right)+(\sqrt{2}+1)\left(\frac{1}{2^{2}}+\frac{2}{2^{4}}+\frac{1}{2^{6}}+\cdots\right) \\
& =1+\frac{\sqrt{2}}{3} .
\end{aligned}
$$


Proposition 6. If $d$ is the intrinsic metric defined in (12) on AS, then

$$
\operatorname{diam}(A S)=\max \{d(A, B) \mid A, B \in A S\}=2 \sqrt{2} .
$$

Moreover, the code representations of $A$ and $B$ satisfying $d(A, B)=2 \sqrt{2}$ are $\widetilde{0} 222 \ldots$ and $\widetilde{1} 222 \ldots$, respectively.

Proof. As seen in Formula (12), to obtain the maximum value of the distances between any two points $A$ and $B$ of $A S, \widetilde{\alpha_{i}}$ and $\widetilde{\beta_{i}}$ must be $\sqrt{2}$ for $i=1,2,3, \ldots$ Then, we get $\widetilde{a_{0}}=0, \widetilde{b_{0}}=1, a_{i}=2$ and $b_{i}=2$ for $i=1,2,3, \ldots$. We also have

$$
\begin{aligned}
d(A, B)=\sum_{i=1}^{\infty} \frac{\widetilde{\alpha}_{i}+\widetilde{\beta}_{i}}{2^{i}} & =\frac{\sqrt{2}+\sqrt{2}}{2}+\frac{\sqrt{2}+\sqrt{2}}{2^{2}}+\frac{\sqrt{2}+\sqrt{2}}{2^{3}}+\frac{\sqrt{2}+\sqrt{2}}{2^{4}}+\cdots \\
& =\sqrt{2}+\frac{\sqrt{2}}{2}+\frac{\sqrt{2}}{2^{2}}+\frac{\sqrt{2}}{2^{3}}+\cdots \\
& =2 \sqrt{2} .
\end{aligned}
$$

\section{Conclusions}

In this paper, we express the intrinsic metrics on two new self-similar (but not strictly self-similar) sets created with different combinations of the Sierpinski gaskets. With a similar way, various self-similar sets can be defined by using the different fractals and the intrinsic metrics on these sets can be formulated.

Author Contributions: The authors contributed equally to this paper.

Funding: This research received no external funding.

Acknowledgments: The authors thank the referees for their valuable comments.

Conflicts of Interest: The authors declare no conflict of interest.

\section{References}

1. Barnsley, M. Fractals Everywhere; Academic Press: San Diego, CA, USA, 1988.

2. Edgar, G. Measure, Topology, and Fractal Geometry; Springer: New York, NY, USA, 2008.

3. Falconer, K. Fractal Geometry: Mathematical Foundations and Applications; Wiley: Hoboken, NJ, USA, 2004.

4. Peitgen, H.O.; Jürgens, H.; Saupe, D. Chaos and Fractals: New Frontiers of Science; Springer-Verlag: New York, NY, USA, 2004.

5. Addison, P.S. Fractals and Chaos: An Illustrated Course; Institute of Physics Publishing: London, UK, 1997.

6. Cristea, L.L.; Steinsky, B. Distances in Sierpinski graphs and on the Sierpinski gasket. Aequationes Math. 2013, 85, 201-219. [CrossRef]

7. Denker, M.; Sato, H. Sierpinski gasket as a Martin boundary II (the intrinsic metric). Publ. Res. Inst. Math. Sci. 1999, 35, 769-794. [CrossRef]

8. Grabner, P.; Tichy, R.F. Equidistribution and Brownian motion on the Sierpinski gasket. Monatshefte für Mathematik 1998, 125, 147-164. [CrossRef]

9. Hinz, A.M.; Schief, A. The average distance on the Sierpinski gasket. Probab. Theory Relat. Fields 1990, 87, 129-138. [CrossRef]

10. Romik, D. Shortest paths in the Tower of Hanoi graph and finite automata. SIAM J. Discret. Math. 2006, 20, 610-622. [CrossRef]

11. Strichartz, R.S. Isoperimetric estimates on Sierpinski gasket type fractals. Trans. Am. Math. Soc. 1999, 351, 1705-1752. [CrossRef]

12. Saltan, M.; Özdemir, Y.; Demir, B. An Explicit Formula of the Intrinsic Metric on the Sierpinski Gasket via Code Representation. Turk. J. Math. 2018, 42, 716-725. [CrossRef]

13. Saltan, M.; Özdemir, Y.; Demir, B. Geodesics of the Sierpinski Gasket. Fractals 2018, 26, 1850024. [CrossRef] 
14. Saltan, M. Some Interesting Code Sets of the Sierpinski Triangle Equipped with the Intrinsic Metric. IJAMAS 2018, 57, 152-160.

15. Saltan, M. Intrinsic Metrics on Sierpinski-Like Triangles and Their Geometric Properties. Symmetry 2018, 10, 204. [CrossRef]

16. Burago, D.; Burago, Y.; Ivanov, S. A Course in Metric Geometry; AMS: Providence, RI, USA, 2001. 